\title{
Agent Affecting Integumentary System
}

National Cancer Institute

\section{Source}

National Cancer Institute. Agent Affecting Integumentary System. NCI Thesaurus. Code C78284.

An agent that exerts a physiologic effect on any anatomic entity that is part of the integumentary system, including skin, nails and hair. 